\title{
Nosocomial Infections: Environmental Sources
}

\author{
Kanokporn Mongkolrattanothai MD, Richard Lampe MD
}

\begin{abstract}
Hall and coworkers studied the rate and burden of bacterial contamination on unused, nonsterile gloves in three different ICUs (medical, surgical and burn ICUs) at a tertiary care medical center in West Texas. A total of 90 glove pair samples were collected consisting of 30 glove pairs from each ICU at the facility. Sterile cotton tipped swabs were used for sampling surfaces for microbial contamination and applied to sterile contact agar plates. Colonies on contact agar plates were further evaluated using MacConkey agar and Luria-Bertani agar media. Colonies which grew on LB agar only were further screened for staphylococci using Staphylococcus medium 110 and for oxacillin resistance using Mueller Hinton agar with $\mathrm{NaCl}$ and $6 \mathrm{microgm} / \mathrm{mL}$ oxacillin.
\end{abstract}

This study revealed growth of gram-positive bacteria in $81.1 \%$ of all glove pairs sampled. Among these bacteria, $36.7 \%$ were oxacillin resistant. Further differentiation of staphylococcal species ( $S$. aureus versus coagulase-negative staphylococci) was not performed. Gram-negative bacteria were not found. The average contamination burden per glove pair was not different among ICUs or patient isolation status. However, the rate of contamination of glove pair samples was relatively lower in the BICU $(66.7 \%)$ compared to the SICU (86.7\%) and the MICU (90\%) $(p=0.044)$. In addition, the rate of glove contamination was lower when samples were obtained from rooms with isolation precautions $(71.1 \%$ vs $88.5 \%, p=0.037)$.

This study was conducted in an attempt to determine whether glove contamination could have a role in the spread of nosocomial infections in the ICUs. As clinicians, we are pleased that gram-negative bacteria were not found on any of the gloves sampled. However, this raises a few questions about the study. First, could we use the results of "bacteria from unused, nonsterile gloves" as an indicator of contamination in ICU environments? Because the gloves are nonsterile, some bacteria could represent manufacturing contaminants. A recent study by Hughes and colleagues ${ }^{1}$ revealed the growth of environmental gram-positive bacteria (e.g., Aerococcus, Micrococcus, Bacillus, etc.) from unused, nonsterile gloves on day 0 when the boxes were just opened. In this study, without data on the contamination rate on day 0 , it is not clear how the results should be interpreted.

We suspect that some bacteria isolated in this study came from the hands of healthcare workers during glove retrieval. However, based on data presented, it would be premature to suggest that the routine use of contact precautions would lower the contamination burden of environmental surfaces, including nonsterile gloves. As the investigators already pointed out in the discussion, it is plausible that a lower contamination burden of the nonsterile gloves in the BICU could be due to the fact that glove boxes are replaced every time a patient is discharged.

Second, could "unused, nonsterile gloves" act as reservoirs and vehicles in the transmission of pathogenic agents? While there is an increasing body of literature that shows the role of the environmental surfaces (e.g., surfaces of medical equipment) in transmission of infections, ${ }^{2}$ the role of unused, nonsterile gloves in the transmission of hospital-associated infections is less clear. A case of disseminated Bacillus cereus infection in the burn unit due to contaminated non-sterile gloves has been reported. ${ }^{3}$ However, universal gloving has the potential to reduce the burden of healthcare-associated pathogens. For example, a recent study by Yin et al. showed that universal gloving was associated with lower rates of hospital-associated infections (e.g., bacteremia and central line associated bloodstream infections) in the pediatric ICU. ${ }^{4}$ Although not all hospital-associated infections can be prevented, it is the responsibility of all healthcare providers to adhere to infection control 
practices including proper hand hygiene to make a safer healthcare environment.

Corresponding Author: Kanokporn Mongkolrattanothai MD Author Contact Information: kanokporn.mongkolrattanothai@ttuhsc.edu

Author Affiliation: Kanokporn Mongkolrattanothai and Richard Lampe are infectious disease specialists in the Department of Pediatrics at TTUHSC Lubbock, TX.

DOI: 10.12746/swrccc2014.0205.052

\section{REFERENCES}

1. Hughes KA, Cornwall J, Theis J, Brooks HJ. Bacterial contamination of unused, disposable non-sterile gloves on a hospital orthopaedic ward. Australasian Med J 2013; 6:331-338.

2. Weber DJ, Anderson D, Rutala WA. The role of the surface environment in healthcare-associated infections. Curr Opin Infect Dis 2013; 26:338-344.

3. Jeurissen A, Weyers L, Cossey V, Muller J, Schuermans A. Dissemination of Bacillus cereus in the burn unit due to contaminated non-sterile gloves. $J$ Hosp Infect 2010; 76:84-95.

4. Yin J, Schweizer ML, Herwaldt LA, Pottinger JM, Perencevich EN. Benefits of universal gloving on hospital-acquired infections in acute care pediatric units. Pediatrics 2013; 131:e1515-e1520. 